\title{
Role of Microbiology Laboratory in Hospital Antimicrobial Stewardship Program: Bringing Backstage to the Apron?
}

\author{
Arash Eatemadi', Sirous Golchinheydari' ${ }^{2}$, Elham Al Risi ${ }^{3}$, \\ Ali Al Reesi ${ }^{1}$ and Ebtihag Elamin Mohamed $^{3}$ \\ ${ }^{1}$ Department of General Medicine, Suhar hospital, Suhar, Sultanate of Oman \\ ${ }^{2}$ Department of Medicine, National University of Science and Technology, Suhar, \\ Sultanate of Oman \\ ${ }^{3}$ Department of Pathology and blood bank, Suhar hospital, Suhar, Sultanate of Oman \\ *Corresponding author
}

\begin{abstract}
A B S T R A C T
\section{Keywords}

Infectious diseases, Antimicrobial Stewardship Program (ASP)

\section{Article Info}

Accepted:

15 January 2021

Available Online:

10 February 2021 desired.

\section{Introduction}

Antimicrobial resistance is related to misuse often overuse -of antimicrobials for both hospitalized and ambulatory patients. There are reasons for inappropriate antimicrobial prescription including lack of a microbiologically supporting data, laboratory mistakes, sending specimens to the laboratory wrongly and over-emphasize on empirical antimicrobial therapy. Antimicrobial stewardship program (ASP) isan interventional package to encourage the
\end{abstract}

Worldwide, all clinicians are daily dealing with infectious diseases in their fields and they rely on microbiology laboratory reports for evidence-based treatment of their patients. Nowadays, antimicrobial stewardship programs (ASPs) are becoming popular in health care systems in order to improve quality of antimicrobial therapy and patient outcome. Active commitment of clinical microbiologists in ASP teams and close collaboration of microbiology laboratory with infectious disease physicians is highly optimal use of antimicrobials in order to achieving the best clinical outcome, with minimal toxicity and impact on following antimicrobial resistance (1). ASP is the cornerstone of antimicrobial policy improvement both in and outside hospitals. From the microbiological view, ASPs diminish antimicrobial resistance, toxicity and side effects, in addition to decrease the rate of clostridioides difficile infection (2).

Because of wide range of infectious diseases and complications, clinical microbiologist has 
todeal with a wide range of clinician. For a successful ASP, Clinical microbiologist and infectious disease physicians should collaborate closely $(3,4,5)$, Although there is wide variation in this cooperation field worldwide (6).

Right diagnosis is basis of effective therapy, including right drug, dose and duration. (7). So, the microbiology laboratory can help clinicians to make an effective therapeutic plan based on right microbiological information. Microbiology laboratory has prominent role in ASP "six D's of antimicrobial stewardship" (Diagnosis, Debridement/drainage, Drug, Dose, Duration, De-escalation) (8).

According to the 2007 IDSA/SHEA guidelines, the membership of clinical microbiologists in ASP team was considered optimal. Their role was described as "the microbiology laboratory plays a critical role in antimicrobial stewardship by providing patient-specific culture and susceptibility data to optimize individual antimicrobial management and by assisting infection control efforts in the surveillance of resistant organisms and in the molecular epidemiologic investigation of outbreaks". The other important mission of microbiology laboratories in ASP described there was providing a local antibiogram panel in order to facilitate making local antimicrobial therapy guidelines. (9)

In a 2016 guideline update, six "to-do" recommendations were described for microbiology laboratory in ASP team: (10)

"We suggest development of stratified antibiograms over solely relying on nonstratified antibiograms to assist ASPs in developing guidelines for empiric therapy (weak recommendation, low-quality evidence)".
"We suggest selective and cascade reporting of antibiotics over reporting of all tested antibiotics (weak recommendation, lowquality evidence)".

"We suggest the use of rapid viral testing for respiratory pathogens to reduce the use of inappropriate antibiotics (weak recommendation, low-quality evidence)".

"We suggest rapid diagnostic testing in addition to conventional culture and routine reporting on blood specimens if combined with active ASP support and interpretation (weak recommendation, moderate-quality evidence)".

"In adults in ICUs with suspected infection, we suggest the use of serial PCT measurements as an ASP intervention to decrease antibiotic use (weak recommendation, moderate-quality evidence)".

"In patients with hematologic malignancy at risk of contracting invasive fungal disease (IFD), we suggest incorporating nonculturebased fungal markers in ASP interventions to optimize antifungal use (weak recommendation, low-quality evidence)". Microbiology laboratories also should provide protocols to clarify how to choose the appropriate test or culture according to the patient signs and symptoms, how to obtain proper clinical specimens, how to send the microbiology laboratory and finally how to interpret microbiology reports. Clear examples in these fields are problems about of blood cultures contaminants and sending unnecessary urine cultures in asymptomatic patients (11).

The Clinical \& Laboratory Standards Institute (CLSI) and European Committee for Antimicrobial Susceptibility Testing (EUCAST) guidelines are updating regularly 
and are essential for microbiology laboratories to provide qualified reports, although there are considerable controversies between them to choose the most appropriate cut-off points which could be challenging (12,13). Therefore, close collaboration between ID physicians and clinical microbiologists is necessary for implementing new breakpoint guidelines $(14,15)$.

In conclusion, we believe that clinical microbiologist plays a crucial role in the ASP through provide fast and accurate microbiology result, especially in the inpatients settingsin order to accelerate diagnosis and management ofinfectious diseases and related complications. Also, we recommend to do regular CME activity and educate clinicians about importance of ASP at the hospital.

\section{References}

Arash Eatemadi, Sirous Golchinheydari, Mohammed Al Reesi, Majid Al Maqbali, Talib Al Maqbali, Aiman Al Wahaibi. Antimicrobial stewardship program in COVID-19 era: Ruin of thereign; Volume 9, Issue 6, JBPR, Nov.-Dec. 2020, due on 10 January2021

Gerding DN. 2001. The search for good antimicrobial stewardship. Jt Comm J Qual Improv27:403-404

Philippe Morency-Potvin, David N. Schwartz, Robert A. Weinstein. Antimicrobial Stewardship: How the Microbiology Laboratory Can Right the Ship. Clin Microb Review. Jan 2017 Volume 30 Issue 1. DOI: 10.1128/CMR.00066-16

Dancer SJ, Varon-Lopez C, Moncayo O, Elston A, Humphreys H. 2015. Microbiology service centralization: a step too far. J Hosp Infect91:292-298. doi:

10.1016/j.jhin.2015.08.017.CrossRefP
ubMedGoogle Scholar

Füsun Can, Onur Karatuna. The Role of Microbiology Laboratory in Promoting Antimicrobial Stewardship. Developments in Emerging and Existing Infectious Diseases. 2017, Pages $115-128$. https://doi.org/10.1016/B978-0-12810477-4.00009-X

Howard P, Pulcini C, Levy Hara G, West RM, Gould IM, Harbarth S, Nathwani D, ESCMID Study Group for Antimicrobial Policies (ESGAP); ISC Group on Antimicrobial Stewardship. 2015. An international cross-sectional survey of antimicrobial stewardship programmes in hospitals. J Antimicrob Chemother 70:1245-1255. doi:10.1093/jac/dku497.

Filice GA, Drekonja DM, Thurn JR, Hamann GM, Masoud BT, Johnson JR. 2015. Diagnostic errors that lead to inappropriate antimicrobial use. Infect Control Hosp Epidemiol 36:949-956. doi:10.1017/ice.2015.113.

Schwartz DN. 2016. Editorial commentary: antimicrobial stewardship in US hospitals: is the cup half-full yet? Clin Infect Dis 63:450-453. doi:10.1093/cid/ciw325.

Dellit TH, Owens RC, McGowan JE, Gerding DN, Weinstein RA, Burke JP, Huskins WC, Paterson DL, Fishman NO, Carpenter CF, Brennan PJ, Billeter M, Hooton TM. 2007. Infectious Diseases Society of America and the Society for Healthcare Epidemiology of America guidelines for developing an institutional program to enhance antimicrobial stewardship. Clin Infect Dis 44:159-177. doi:10.1086/510393.

Barlam TF, Cosgrove SE, Abbo LM, MacDougall C, Schuetz AN, Septimus EJ, Srinivasan A, Dellit TH, FalckYtter YT, Fishman NO, Hamilton CW, Jenkins TC, Lipsett PA, Malani PN, 
May LS, Moran GJ, Neuhauser MM, Newland JG, Ohl CA, Samore MH, Seo SK, Trivedi KK. 2016. Executive summary: implementing an antibiotic stewardship program: guidelines by the Infectious Diseases Society of America and the Society for Healthcare Epidemiology of America. Clin Infect Dis 62:1197-1202. doi:10.1093/cid/ciw217.

Australian Commission on Safety and Quality in Health Care. 2011. Antimicrobial stewardship in Australian hospitals, January 2011 ed. Australian Commission on Safety and Quality in Health Care, Sydney, NSW, Australia: https://www.safetyandquality.gov.au/o ur-work/healthcare-associatedinfection/antimicrobialstewardship/book/.

Marchese A, Esposito S, Barbieri R, Bassetti M, Debbia E. 2012. Does the adoption of EUCAST susceptibility breakpoints affect the selection of antimicrobials to treat acute community-acquired respiratory tract infections? BMC Infect Dis 12:181. doi:10.1186/14712334-12-181.

Wolfensberger A, Sax H, Weber R, Zbinden R, Kuster SP, Hombach M. 2013. Change of antibiotic susceptibility testing guidelines from CLSI to EUCAST: influence on cumulative hospital antibiograms. PLoS One 8:e79130. doi:10.1371/journal.pone.0079130.

Ginocchio CC. 2002. Role of NCCLS in antimicrobial susceptibility testing and monitoring. Am J Health Syst Pharm 59(8 Suppl 3): S7-S11.

Clinical and Laboratory Standards Institute. 2015. M100-S25 performance standards for antimicrobial susceptibility testing; twenty-fifth informational supplement. Clinical and Laboratory Standards Institute, Wayne, PA.

\section{How to cite this article:}

Arash Eatemadi, Sirous Golchinheydari, Elham Al Risi, Ali Al Reesi and Ebtihag Elamin Mohamed. 2021. Role of Microbiology Laboratory in Hospital Antimicrobial Stewardship Program: Bringing Backstage to the Apron? Int.J.Curr.Microbiol.App.Sci. 10(02): 1569-1572. doi: https://doi.org/10.20546/ijcmas.2021.1002.186 